\title{
Effects of starvation on the olfactory responses of the blood- sucking bug Rhodnius prolixus
}

\author{
Carolina E. Reisenman ${ }^{1}$, Yan Lee ${ }^{1}$, Teresa Gregory ${ }^{1}$, and Pablo G. Guerenstein ${ }^{2}$ \\ ${ }^{1}$ Department of Neuroscience, University of Arizona, Tucson, AZ 85721-0077, USA \\ ${ }^{2}$ CICyTTP-CONICET \& UNER, Entre Ríos, Argentina
}

\section{Abstract}

Blood-sucking insects use olfactory cues in a variety of behavioral contexts, including hostseeking and aggregation. In triatomines, which are obligated blood-feeders, it has been shown that the response to $\mathrm{CO}_{2}$, a host-associated olfactory cue used almost universally by blood-sucking insects, is modulated by hunger. Host-finding is a particularly dangerous task for these insects, as their hosts are also their potential predators. Here we investigated whether olfactory responses to host-derived volatiles other than $\mathrm{CO}_{2}$ (nonanal, a-pinene and (-)-limonene), attractive odorant mixtures (yeast volatiles), and aggregation pheromones (present in feces) are also modulated by starvation in the blood-sucking bug Rhodnius prolixus. For this, the responses of both non-starved and starved insects were individually tested at the beginning of the scotophase using a dual-choice "T-shaped" olfactometer, in which one of its arms presented odor-laden air and the other arm presented odorless air. We found that the response of non-starved insects towards host-odorants and odorant mixtures was odor-dependent: insects preferred the odor-laden arm of the maze when tested with a-pinene, the odorless arm of the maze when tested with (-)-limonene, and distributed at random when tested with yeast volatiles or nonanal. In contrast, starved insects significantly preferred the odor-laden arm of the maze when tested with host-odorants or yeast volatiles. When tested with aggregation pheromones, non-starved insects distributed randomly between the arms of the maze, while starved insects preferred the odorless arm of the maze; insects that were even more starved (8-9 weeks post-ecdysis) significantly preferred the odor-laden arm of the maze. We postulate that this odor- and starvation-dependent modulation of sensory responses has a high adaptive value, as it minimizes the costs and risks associated with the associated behaviors. The possible physiological mechanisms underlying these modulatory effects are discussed.

\section{Keywords}

triatomine; Rhodnius prolixus; olfaction; behavior; sensory modulation

\section{INTRODUCTION}

Blood-sucking insects such as mosquitoes, ticks and triatomines are vectors of a myriad of human and animal diseases (Lehane 2005). Host-searching and location are particularly

\footnotetext{
(C) 2013 Elsevier Ltd. All rights reserved.

Correspondence should be addressed to Dr. Carolina E. Reisenman, Department of Neuroscience, University of Arizona, PO Box 210077, Tucson, AZ 85721-0077, USA., carolina@ neurobio.arizona.edu, Phone: 1-520-621-6631.

Publisher's Disclaimer: This is a PDF file of an unedited manuscript that has been accepted for publication. As a service to our customers we are providing this early version of the manuscript. The manuscript will undergo copyediting, typesetting, and review of the resulting proof before it is published in its final citable form. Please note that during the production process errors may be discovered which could affect the content, and all legal disclaimers that apply to the journal pertain.
} 
dangerous activities for blood-sucking insects, as their hosts are also potential predators. Accordingly, it has been shown that mosquitoes and triatomines only search for a host when they are hungry and ready to take a blood meal (Bodin et al. 2009a; Bodin et al. 2009b; Takken et al. 2001). Different from mosquitoes, where only females feed on blood, triatomines of all life stages are obligated blood-feeders and therefore are highly exposed to predation.

Triatomines search for food during the night using mostly thermal (Flores and Lazzari 1996) and olfactory cues such as L-lactic acid, pyruvic acid, short-chain carboxylic acids, aldehydes, pyridine, furan, terpenes, ammonia, amine (Barrozo and Lazzari 2004a; Barrozo and Lazzari 2004b; Guerenstein and Guerin 2001; Guerenstein and Lazzari 2009; Taneja and Guerin 1997) and $\mathrm{CO}_{2}$ (Barrozo and Lazzari 2004b; Barrozo et al. 2004; Guerenstein et al. 1995; Taneja and Guerin 1995). During the day triatomines are mostly inactive and remain aggregated inside dark refuges. The aggregation behavior of insects is mediated by thigmotaxis and by volatiles present in their feces (Lorenzo Figueiras et al. 1994), which serve as chemical markers of refuges (Lorenzo and Lazzari 1996).

In triatomines, it has been shown that the response to heat and $\mathrm{CO}_{2}$, which respectively act as short- and long-distance host-associated cues (Núñez 1982), as well as the motivation to feed, are modulated by starvation. While starved insects responded positively to these two orientation cues, non-starved insects remained indifferent to heat and were repelled by $\mathrm{CO}_{2}$ (Bodin et al. 2009a; Bodin et al. 2009b). $\mathrm{CO}_{2}$ is produced in large quantities by human breath [ $4.5 \%$ vs. $0.04 \%$ normally present in the atmosphere (Lehane 2005)] and is an important, ubiquitous host-derived olfactory cue used by blood-sucking insects (Lehane 2005). The purpose of this work therefore was to investigate the modulatory effects of starvation in the olfactory responses of triatomine bugs towards host-derived odorants other than $\mathrm{CO}_{2}$, attractive odorant mixtures which contain many host-derived odorants (yeast volatiles, Guerenstein et al. 1995), and towards odors involved in mediating other olfactorydriven behaviors such as aggregation (Lorenzo Figueiras et al. 1994). Our initial prediction was that starvation would decrease responses towards aggregation cues but would increase responses towards host-derived cues (Bodin et al. 2009a; Bodin et al. 2009b), as starved insects should be motivated to feed. Experiments were conducted with the triatomine species Rhodnius prolixus, one of the most important vectors of Chagas disease in Central America and in northern South America, and a classical model in studies of insect physiology and behavior.

\section{MATERIALS AND METHODS}

\section{Animals}

Insects were fed weekly in the laboratory with bovine blood (with $2 \%$ sodium citrate added as anticoagulant) supplemented with ATP $(100 \mathrm{mM})$ using an artificial feeder apparatus (Núñez and Lazzari 1990). Although insects normally acquire necessary symbionts by coprophagy (Lehane 2005), the symbiont Rhodococcus rhodnii was added to the blood approximately once a month to ensure colonization and therefore adequate nutrition (Baines 1956). About 4-7 days after eclosion to the third or fourth instar stage, insects were separated (and kept unfed) until experiments were conducted. Experiments were conducted with two groups of insects, non-starved and starved. Non-starved insects were used $10 \pm 2$ days (in the case of $3^{\text {rd }}$ instar nymphs) or $14 \pm 1$ days (in the case of $4^{\text {th }}$ instar nymphs) after eclosion to the $3^{\text {rd }}$ or $4^{\text {th }}$ stage, respectively. Starved insects were used 4-5 weeks or 6-7 weeks after eclosion to the $3^{\text {rd }}$ and $4^{\text {th }}$ instar, respectively. Additionally, $4^{\text {th }}$ instar nymphs starved during 8-9 weeks after eclosion were tested with aggregation pheromones and (-)limonene. Because insects were offered food once a week, in all cases the time elapsed since the last meal was at least seven more days than the times indicated in the previous sentences 
(e.g. non-starved $3^{\text {rd }}$ instar insects were offered food $17 \pm 2$ days before experiments were conducted).

\section{Experimental procedure}

The olfactory responses of insects were tested using a dual-choice "T-shaped" olfactometer $(13.1 \mathrm{~cm} \times 17.6 \mathrm{~cm}$; Fig. 1 and Supp. Video 1$)$ modified from one previously published (Ortiz and Molina 2010). Several T-mazes were constructed using tygon tubing (VMR International Brand, Thermo Scientific; external diameters: 1.3 and $1.6 \mathrm{~cm}$ for the arms and leg of the T, respectively), which allowed us to change mazes for clean ones every 3-4 tests. The open ends of the arms of the "T" were each connected to a stimulus container using tygon tubing ( $4 \mathrm{~mm}$ internal diameter). Charcoal-filtered air was delivered to each of the two stimulus containers (odor-laden and control) and adjusted to 0.5 LPM using two independent mass flow controllers (Alicat Scientific, Tucson, Arizona, USA). Thus, at the insect release site (see below), air flow was ca. 1 LPM (measured with a digital flow-meter, Alicat Scientific, Tucson, Arizona, USA). About 5-10 minutes before being tested, each individual insect was placed in an open-top and mesh-bottom cylindrical holder $(4.5 \mathrm{~cm} \times 1.5 \mathrm{~cm})$ constructed using tygon tube. Each test startedwhen the open-top of the insect holder was carefully slid into the open end of the long arm of the "T" (see Fig. 1). Thus, upon being released, insects could walk upwind towards the "decision point" (intersection of the short and long arms of the "T") and turn towards either the odor-laden or the odorless arm of the maze (Fig. 1, Supp. Video 1). Insects that made a choice usually reached the distal end of the arm but could not walk any further, precluded by the reduction in tube size (Fig. 1, Supp. Video 1). Occasionally, insects switched between the odor-laden and the odorless arm, but only the first choice of each insect was recorded. A choice was considered as such only if the insect walked past at least 1.5 times its body length into the arm, orienting upwind (Supp. Video 1). Each test lasted a maximum of eight minutes, and each insect was used only once. Results from an individual test session were discarded if less than $20 \%$ of the insects made a choice between the arms of the maze. Tests in which the insect was visibly startled upon being released were not considered. The position of the control and the test arms was switched every two tests to control for positional asymmetries. In one experimental series with insects starved during 8-9 weeks one arm of the maze presented aggregation pheromones, while the other offered (-)-limonene; the position of the odors was also switched every two tests. The T-mazes were replaced by clean ones every 3-4 tests; Tmazes were cleaned with $70 \%$ ethanol and allowed to air-dry before reusing. A total of 483 individual insects were tested, $77.8 \%$ of which made a choice between the arms of the maze. Although both $3^{\text {rd }}$ and $4^{\text {th }}$ instar insects were used throughout experiments, insects of the same stage were used for each odor tested (tests with yeast volatiles, a-pinene and nonanal always were conducted with $3^{\text {rd }}$ instar insects; tests with (-)-limonene and feces always were conducted with $4^{\text {th }}$ instar insects). In all cases insects were tested during the $1^{\text {st }}$ to $3^{\text {rd }}$ hour of their scotophase under low light permissive conditions provided with a dimmed 25 $\mathrm{W}$ red incandescent light.

\section{Odors and odor sources}

We tested the olfactory responses of larvae of $R$. prolixus towards single synthetic hostderived odorants, yeast volatiles (Guerenstein et al. 1995), and dry feces, the source of aggregation pheromones (Lorenzo Figueiras et al. 1994). Yeast volatiles provide a complex odorant mixture (Ames and Leod 1985) which includes many host-derived odorants and $\mathrm{CO}_{2}$. The following asynthetic host-derived odorants (Guerenstein and Guerin 2001, Syed and Leal 2009, Guerenstein 1999), all diluted in mineral oil (Sigma-Aldrich, St. Louis, USA), were used: $0.006 \%$ (vol/vol) nonanal (Fluka, Steinheim, Switzerland, CAS \#: 124-19-6, 97\% pure); $0.05 \%$ a-pinene (Aldrich, CAS \# 80-56-8, 98\% pure); and 0.05\% (-)limonene (Fluka, Steinheim, Switzerland, CAS \# 5989-54-8, 99\% pure, sum of 
enantiomers). Concentrations of odorants were chosen based on previously published data (Ortiz and Molina 2010) and preliminary tests (not shown). Fifty microliters of odorant solution were loaded in a piece of filter paper $(1 \mathrm{~cm} \times 1 \mathrm{~cm})$ and inserted into a $5 \mathrm{ml}$ glass syringe right before tests; control syringes had a piece of filter paper loaded with $50 \mu l$ of mineral oil. Syringes and paper filters with synthetic odorants were replaced by fresh ones every $2-3$ tests. Yeast cultures were prepared in a $260 \mathrm{ml}$ glass flask approximately $15-20$ minutes before tests started by mixing $10 \mathrm{ml}$ of $25-30^{\circ} \mathrm{C}$ tap water, $4 \mathrm{~g}$ of sucrose, and $1 \mathrm{~g}$ of dry baker's yeast. Control flasks contained $10 \mathrm{ml}$ of water at the same temperature and 4 $\mathrm{g}$ of sucrose. Each flask had two tygon tubes connected to its tight-fitting lid: one tube directed clean filtered air into the flask, and the other tube provided air (either carrying yeast volatiles or control volatiles) to one of the short arms of the T-maze (Fig. 1B). Yeast cultures provided volatiles throughout the duration of an experimental session (ca. 1.5-2 hours). As a source of aggregation pheromones, pieces of filter paper (ca. $1 \mathrm{~cm} \times 1 \mathrm{~cm}$ each) impregnated with 24 hour-old dry feces from $4^{\text {th }}$ and $5^{\text {th }}$ larvae of $R$. prolixus were used (Lorenzo Figueiras et al. 1994). Fecal droplets were obtained by slightly compressing the terminal segment of the abdomen of insects with fine tweezers; insects often defecated spontaneously when held with tweezers. Feces obtained in this way were shown to be effective in inducing aggregation in triatomines (Reisenman et al. 2000). Syringes and filter papers with feces were replaced by fresh ones every 3-4 tests. Control tests, in which insects were exposed to two odorless air currents, were regularly conducted to test for positional asymmetries.

\section{Statistics}

One-tailed binomial tests were conducted to test whether the proportion of insects choosing the arm of the olfactometer with clean air or the odor-laden arm of the olfactometer statistically differed from the 50\% random expectation (Zar 1999). The effect of starvation for a given odorant was analyzed using Fisher exact tests (Zar 1999). Results were considered significant if $\mathrm{p}<0.05$.

\section{RESULTS}

The olfactory responses of insects $(\mathrm{N}=329)$ are shown in Figure 2. As expected, insects exposed to two control clean air currents choose randomly between the two arms of the olfactometer (binomial test, $\mathrm{p}>0.05$; only starved insects were tested with two clean air currents). Starved insects showed statistically significant oriented responses towards yeast volatiles, (-)-limonene, nonanal and a-pinene (Fig. 2, gray bars; binomial tests, $p<0.05$ in all cases). Non-starved insects also showed oriented responses towards a-pinene, but avoided (-)-limonene, with 70\% of insects choosing the control (odorless) arm of the maze (binomial tests, in both cases $p<0.05$, Fig. 2). Non-starved insects tested with yeast volatiles and nonanal distributed randomly between the two arms of the maze (binomial tests, $\mathrm{p}>0.05$ in both cases; Fig. 2). Comparison of the responses of non-starved and starved insects revealed that the effects of starvation were significant for tests conducted with yeast volatiles, nonanal and limonene (Fisher exact tests, $p<0.05$ in all cases) but not for tests with a-pinene (Fisher exact test, $\mathrm{p}>0.05$ ).

Non-starved insects tested with aggregation pheromones choose randomly between the two arms of the maze (binomial test, $p>0.05$; Fig. 2), while starved insects significantly preferred the odorless arm of the maze (binomial test, Fig. 2, p<0.05). In contrast, insects that were more starved (tested 8-9 weeks postecdysis) significantly preferred the arm of the maze laden with the pheromone odor (Fig. 2, p<0.05). Differences between the responses of starved insects and insects starved for 8-9 weeks postecdysis were statistically significant (Fisher exact test, $\mathrm{p}<0.05$ ). Differences between the responses of non-starved and starved 
insects, as well as those of non-starved vs. more starved insects, were not statistically different (Fisher exact tests, $\mathrm{p}>0.05$ in both cases).

We also tested whether insects starved during 8-9 weeks oriented towards host odors. As expected, we found that insects significantly preferred the arm of the maze laden with the host odor (only (-)-limonene was tested; binomial test, $\mathrm{p}<0.05$; Fig. 2). The responses of insects were statistically different from those of non-starved insects (Fisher exact test, $\mathrm{p}<0.05$ ) but did not differed from the responses of insects starved during 4-5 weeks (Fisher exact test, $\mathrm{p}>0.05)$. We next conducted tests in which one arm of the maze offered a host odor [(-)-limonene] and the other arm offered aggregation pheromones. Although as predicted, a larger proportion of insects preferred the arm of the maze with the host-odor $(57.4 \%, \mathrm{n}=47)$, differences were not statistically significant (binomial test, $\mathrm{p}>0.05)$.

\section{DISCUSSION}

We found that starvation differentially affects the olfactory responses of $R$. prolixus towards host-derived olfactory cues and aggregation pheromones. As expected, starved (i.e. hungry) insects positively oriented towards all the host-derived odorants tested and yeast volatiles. The response of non-starved insects towards host odorants further depended on the chemical identity of the odorant tested. Non-starved insects were attracted to a-pinene, choose the odorless arm of the maze when tested with (-)-limonene, and distributed at random when tested with nonanal or yeast volatiles [which is a complex mixture that includes $\mathrm{CO}_{2}$ (Ames and Leod 1985; Guerenstein et al. 1995)] (Fig. 2). These results are in line with previous findings showing that $\mathrm{CO}_{2}$ (a long-range host-derived olfactory cue), but not heat (a shortrange host-derived cue), is not attractive to non-starved insects (Bodin et al. 2009a). Here we also report, for the first time, that terpenes of vertebrate origin such as (-)-limonene and apinene are attractive to triatomines (Fig. 2). These two odorants have been reported as volatile constituents of chicken feathers and sheep wool (Guerenstein 1999). Limonene and pinene were also respectively reported as volatile constituents of human urine (Zlatkis and Liebich 1971) and human effluvia (Ellin et al. 1974).

When presented with aggregation pheromones, the responses of insects also depended on the starvation status (Fig. 2). Non-starved insects distributed at random between the arms of the maze, and starved insects, as expected, avoided the odor-laden arm of the maze.

Interestingly, insects that were even more starved (tested 8-9 weeks postecdysis) significantly preferred the arm of the maze laden with aggregation pheromones (Fig. 2). The biological significance of this modulation of the response toward aggregation pheromones with prolonged starvation (8-9 weeks) may be that insects, depleted of resources, would have a strong tendency to remain aggregated in refuges unless there is a clear signal that a host is available. Indeed, we found that insects with prolonged starvation oriented towards the host odor (-)-limonene (Fig. 2). Moreover, in experiments in which insects with prolonged starvation were simultaneously tested with aggregation pheromones and a hostodor [(-)-limonene], a larger proportion of insects choose the arm of the maze laden with the host-odor. In the case of non-starved insects, which distributed at random, it is possible that the modulatory effect of starvation in the response towards aggregation pheromones is further modulated by the time of the day, as less-starved nymphs tend to search for food instead of refuges at the beginning of the scotophase (which is the time of the day during which insects were tested in our experiments). This is supported by the finding that insects older than six days postecdysis disperse and aggregate at the beginning and end of the scotophase, respectively (Lorenzo Figueiras et al. 1994). In any case, our results demonstrate that the response towards aggregation pheromones is modulated by the starvation status, even when insects were tested during their main activity period (early scotophase). The effect of starvation in the responses towards aggregation pheromones has 
also been studied in other blood-sucking insects. For instance, it has been shown that bed bugs oriented towards aggregation pheromones regardless of the nutritional state when tested during their inactivity period (Weeks et al. 2013), but that responses towards aggregation pheromones tended to decrease with increased starvation (Olson et al. 2009).

We have not found major differences with previous studies in triatomines (Bodin et al. 2009a) that used a locomotor compensation device to test the responses of insects towards $\mathrm{CO}_{2}$ (in our experiments insects could walk freely on the T-maze). The fact that similar results were obtained using different experimental designs emphasizes the biological significance of these findings, that is, that the starvation state has profound effects on the olfactory behavior of triatomines.

Our experiments also show that the effects of starvation in the olfactory responses of insects also depend on the biological meaning of the olfactory signal (host-odors vs. aggregation pheromones). Olfactory responses to odorants with different behavioral meanings also are differentially modulated by the time of day in triatomines: in starved insects, responses to host-derived odorants and aggregation pheromones were shown to be stronger at the beginning and end of the scotophase, respectively (Barrozo et al. 2004; Bodin et al. 2008).

The mechanisms mediating the effects of hunger in olfactory responses and food-search have been mainly investigated in the fruit fly Drosophila melanogaster (Marella et al. 2012; Root et al. 2011; Sengupta 2013). For example, while insulin and short neuropeptide F modulate olfactory receptor cell sensitivity (Root et al. 2011), dopamine modulates sugar acceptance (Marella et al. 2012), and allatostatin-A inhibits or limit feeding (Hergarden et al. 2012). Other neuropeptides such as tachykinins have been implicated in signaling feeding status in other insect species (Nässel and Winther 2010). In triatomines, it has been shown that an unidentified factor in the haemolymph mediates the modulation of olfactory responses by starvation (Bodin et al. 2009a). The identity of this factor(s), as well as the neural mechanisms involved in mediating sensory modulation, remain to be investigated

In summary, our results show that feeding status has profound effects in modulating the olfactory seeking behavior of triatomines, with odorants with different behavioral meanings having differential behavioral effects. Not only this modulation of sensory responses has a high adaptive value, but also has important implications for parasite disease transmission.

\section{Supplementary Material}

Refer to Web version on PubMed Central for supplementary material.

\section{Acknowledgments}

Authors are indebted to Ellen Dotson (CDC) for providing insects to start a colony of $R$. prolixus and to Kristin Cobb (CDC) for routinely providing cultures of $R$. rhodnii, to John G. Hildebrand for his continuing support and advice, to two anonymous reviewers for comments and suggestions that greatly improved this manuscript, and to Bianca Demara for help rearing insects. This work was partially supported by an RO3 NIH grant (1R03AI078430-01) to John G. Hildebrand.

\section{References}

Ames JM, Leod GM. Volatile components of a yeast extract composition. Journal of Food Science. $1985 ; 50: 125-131$.

Baines S. The role of the symbiotic bacteria in the nutrition of Rhodnius prolixus (Hemiptera). The Journal of Experimental Biology. 1956; 33:533-541. 
Barrozo RB, Lazzari CR. Orientation behaviour of the blood-sucking bug Triatoma infestans to shortchain fatty acids: synergistic effect of (L)-lactic acid and carbon dioxide. Chemical Senses. 2004a; 29:833-841. [PubMed: 15574819]

Barrozo RB, Lazzari CR. The response of the blood-suckin bug Triatoma infestans to carbon dioxide and other host odours. Chemical Senses. 2004b; 29:319-329. [PubMed: 15150145]

Barrozo RB, Minoli SA, Lazzari CR. Circadian rhythm of behavioural responsiveness to carbon dioxide in the blood-sucking bug Triatoma infestans (Heteroptera: Reduviidae). Journal of Insect Physiology. 2004; 50:249-254. [PubMed: 15019528]

Bodin A, Barrozo RB, Couton L, Lazzari CR. Temporal modulation and adaptive control of the behavioural response to odours in Rhodnius prolixus. Journal of Insect Physiology. 2008; 54:13431348. [PubMed: 18692504]

Bodin A, Vinauger C, Lazzari CR. Behavioural and physiological state dependency of host seeking in the blood-sucking insect Rhodnius prolixus. Journal of Experimental Biology. 2009a; 212:23862393. [PubMed: 19617431]

Bodin A, Vinauger C, Lazzari CR. State-dependency of host-seeking in Rhodnius prolixus: the postecdysis time. Journal of Insect Physiology. 2009b; 55:574-579. [PubMed: 19418597]

Ellin RI, Farrand RL, Oberst FW, Crouse CL, Billups NB, Koon WS, Musselman NP, Sidell FR. An apparatus for the detected and quantitation of volatile human effluents. Journal of Chromatography A. 1974; 100:137-152.

Flores G, Lazzari CR. The role of the antennae in Triatoma infestans: orientation towards thermal sources. Journal of Insect Physiology. 1996; 42:443-440.

Guerenstein P, Guerin P. Olfactory and behavioural responses of the blood-sucking bug Triatoma infestans to odours of vertebrate hosts. The Journal of Experimental Biology. 2001; 204:585-597. [PubMed: 11171309]

Guerenstein, PG. PhD Thesis. University of Neuchâtel; Switzerland: 1999. Sensory and behavioural responses of Triatoma infestans to host and conspecific odours.

Guerenstein PG, Lazzari CR. Host-seeking: How triatomines acquire and make use of information to find blood. Acta Tropica. 2009; 110:148-158. [PubMed: 18983971]

Guerenstein PG, Lorenzo MG, Núñez JA, Lazzari CR. Baker's yeast, an attractant for baiting traps for Chagas' disease vectors. Experientia. 1995; 51:834-837. [PubMed: 7649243]

Hergarden AC, Tayler TD, Anderson DJ. Allatostatin-A neurons inhibit feeding behavior in adult Drosophila. Proceedings of the National Academy of Sciences USA. 2012; 109:3967-3972.

Lehane, M. The biology of blood-sucking in insects. New York: Cambridge University Press; 2005. p. 321

Lorenzo Figueiras AN, Kenigsten A, Lazzari CR. Aggregation in the haematophagous bug Triatoma infestans: chemical signals and temporal pattern. Journal of Insect Physiology. 1994; 40:311-316.

Lorenzo MG, Lazzari CR. The spatial pattern of defaecation in Triatoma infestans and the role of faeces as a chemical mark of the refuge. Journal of Insect Physiology. 1996; 42:903-907.

Marella S, Mann K, Scott K. Dopaminergic modulation of sucrose acceptance behavior in Drosophila. Neuron. 2012; 73:941-950. [PubMed: 22405204]

Nässel DR, Winther ÅME. Drosophila neuropeptides in regulation of physiology and behavior. Progress in Neurobiology. 2010; 92:42-104. [PubMed: 20447440]

Núñez JA. Food source orientation and activity in Rhodnius prolixus Stäl (Hemiptera: Reduviidae). Bulletin of Entomological Research. 1982; 72:253-262.

Núñez JA, Lazzari CR. Rearing of Triatoma infestans Klug (Hemiptera: Reduviidae) in the absence of a live host. I. Some factors affecting the artificial feeding. Journal of Applied Entomology. 1990; 109:87-92.

Olson JF, Moon RD, Kells SA. Off-host aggregation behavior and sensory basis of arrestment by Cimex lectularius (Heteroptera: Cimicidae). Journal of Insect Physiology. 2009; 55:580-587. [PubMed: 19418598]

Ortiz MI, Molina J. Preliminary evidence of Rhodnius prolixus (Hemiptera: Triatominae) attraction to human skin odour extracts. Acta Tropica. 2010; 113:174-179. [PubMed: 19879853] 
Reisenman CE, Lorenzo Figueiras AN, Giurfa M, Lazzari CR. Interaction of visual and olfactory cues in the aggregation behaviour of the haematophagous bug Triatoma infestans. Journal of Comparative Physiology A. 2000; 186:961-968.

Root CM, Ko KI, Jafari A, Wang JW. Presynaptic facilitation by neuropeptide signaling mediates odor-driven food search. Cell. 2011; 145:133-144. [PubMed: 21458672]

Sengupta $P$. The belly rules the nose: feeding state-dependent modulation of peripheral chemosensory responses. Current Opinion in Neurobiology. 2013; 23:68-75. [PubMed: 22939570]

Syed Z, Leal WS. Acute olfactory response of Culex mosquitoes to a human- and bird-derived attractant. Proceedings of the National Academy of Sciences. 2009; 106:18803-18808.

Takken W, van Loon JJ, Adam W. Inhibition of host-seeking response and olfactory responsiveness in Anopheles gambiae following blood feeding. Journal of Insect Physiology. 2001; 47:303-310. [PubMed: 11119776]

Taneja J, Guerin P. Oriented responses of the triatomine bugs Rhodnius prolixus and Triatoma infestans to vertebrate odours on a servosphere. Journal of Comparative Physiology A. 1995; 176:455-464.

Taneja J, Guerin P. Ammonia attracts the haematophagous bug Triatoma infestans: behavioural and neurophysiological data on nymphs. Journal of Comparative Physiology A. 1997; 181:21-34.

Weeks ENI, Logan JG, Birkett MA, Pickett JA, Cameron MM. Tracking bed bugs (Cimex lectularius): a study of the effect of physiological and extrinsic factors on the response to bed bug-derived volatiles. The Journal of Experimental Biology. 2013; 216:460-469. [PubMed: 22996447]

Zar, JH. Biostatistical analysis. Upper Saddle River, NJ: Prentice-Hall; 1999. p. 944

Zlatkis A, Liebich HM. Profile of volatile metabolites in human urine. Clinical Chemistry. 1971; 17:592-594. [PubMed: 5556886] 
- We investigated the effect of hunger in the odor responses of Rhodnius prolixus.

- Starved insects positively oriented towards host-derived odorants.

- The response of non-starved insects was variable.

- Only very starved insects responded to aggregation pheromones. 


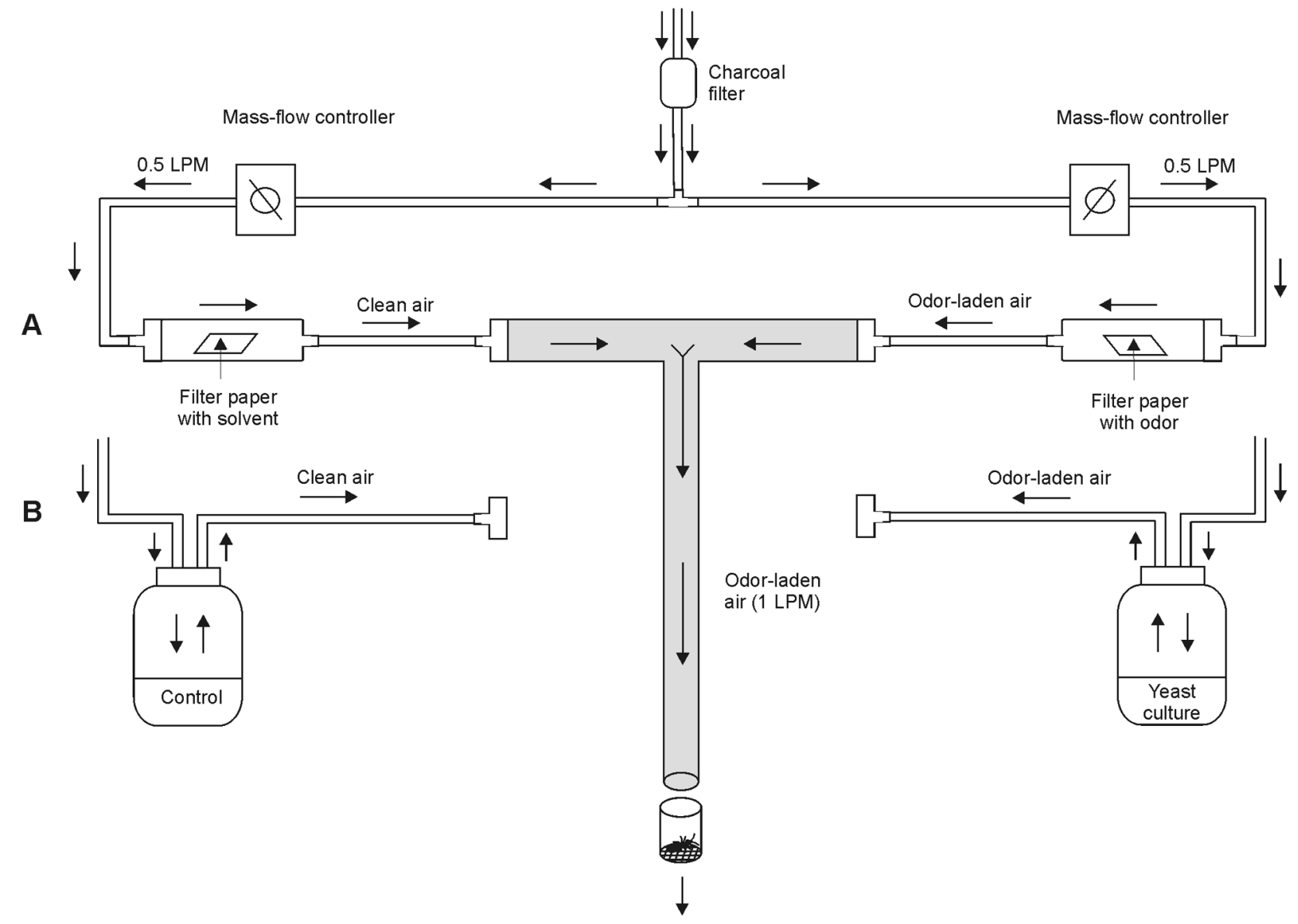

Figure 1.

Schematic illustration of the behavioral set-up used to study the olfactory responses of insects using synthetic odorants (A). B shows the odor configuration used for tests with yeast volatiles (glass flasks with yeast cultures). Arrows indicate the air flow direction. 


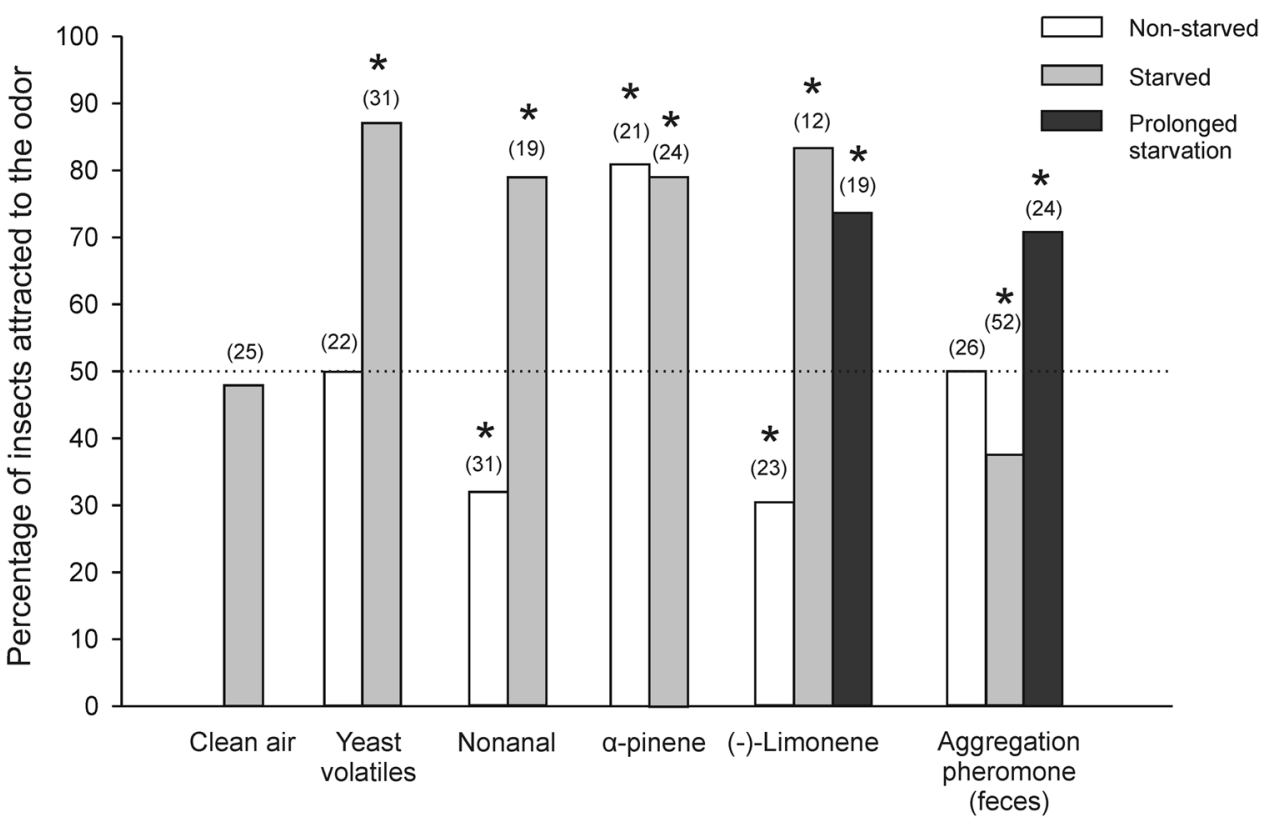

Figure 2.

Olfactory responses of non-starved (white bars) and starved (light gray bars) insects towards two clean air currents, host-derived odorants (yeast volatiles, $0.05 \%$ (-)-limonene, $0.006 \%$ nonanal, $0.05 \%$ a-pinene) and con-specific aggregation pheromones. Only starved insects were tested with two clean currents. Two additional groups of $4^{\text {th }}$ instar insects starved during 8-9 weeks were tested with aggregation pheromones and $0.05 \%$ (-)-limonene (black bars). Data indicates the percentage of insects attracted to the arm of the maze with the odor (or to a reference arm in the case of insects tested with two clean air currents). The number of insects tested for each combination of odor and starvation state is indicated above each bar. Asterisks indicate that for the proportion of insects choosing the odor-laden arm of the maze was statistically different from the $50 \%$ random expectation (binomial tests, $\mathrm{p}<0.05$ ) for a given odor-starvation state. 